\title{
Modelling human actions on lightweight structures: experimental and numerical developments
}

\author{
S. Živanović , a $^{1}$ \\ ${ }^{1}$ University of Warwick, School of Engineering, Coventry CV4 7AL, United Kingdom
}

\begin{abstract}
This paper presents recent, numerical and experimental, developments in modelling dynamic loading generated by humans. As modern structures with exposure to human-induced loading, such as footbridges, building floors and grandstands, are becoming ever lighter and more slender, they are increasingly susceptible to vibration under human-induced dynamic excitation, such as walking, jumping, running and bobbing, and their vibration serviceability assessment is often a deciding factor in the design process. While simplified modelling of the human using a harmonic force was sufficient for assessment of vibration performance of more robust structures a few decades ago, the higher fidelity models are required in the contemporary design. These models are expected not only to describe both temporal and spectral features of the force signal more accurately, but also to capture the influence, psychological and physiological, of human-structure and human-human interaction mechanisms on the human kinematics, and consequently on the force generated and the resulting vibration response. Significant advances have been made in both the research studies and design guidance. This paper reports the key developments and identifies the scope for further research.
\end{abstract}

\section{Introduction}

Most structures are made to accommodate people in a variety of settings, for example as living spaces (residential buildings), working environments (office buildings), entertainment venues (music halls, dance floors and sports stadia), fitness centres (gymnasiums), footpaths over rivers, roads and other "obstacles" (bridges), and so on. The loading scenarios considered relevant for structural design a few decades ago regularly included the self-weight of structural and non-structural elements, environmental loads (caused, for example, by wind, snow, earthquakes, temperature changes, and similar) and other loads specific for the intended purpose of the structure (such as vehicle or train traffic over road and rail bridges, respectively). Loading by humans was often neglected or, if the structure accommodated a large number of people, considered as static weight only. However, humans are active creatures, and as they move they generate a dynamic force that is induced into the supporting structure. For long time this dynamic loading was considered insignificant and it did not feature in the design. In 1978 the British Standards Institution published what is believed to be the first vibration serviceability guidance for footbridges BS5400 [1]. A walker was modelled simply as a harmonic force crossing the bridge at a constant speed and having a frequency that matches one of structural resonances. This approach worked well for traditional structures designed with large factors of safety that were typically heavier and stiffer than they would be if designed using the contemporary design guidance. Although general advances in design, including optimisation of material use, started to result in lighter, less damped and longer-span structures several decades ago, it was only after highly publicised vibration serviceability problems of the Solférino Bridge in Paris [2] and the Millennium Bridge in London [3] under crowd of walkers on the opening days in 1999 and 2000, respectively, that modelling human actions drew attention of researchers worldwide and strongly influenced the landscape of vibration serviceability assessment in years to come.

While the problem of excessive vibration is being highlighted by bold design of contemporary structures aiming to minimise use of construction materials as well as to make strong aesthetic impact (Figure 1), both of which usually lead to light and slender solutions that are easier to bring into motion by human actions, the research into past vibration serviceability failures showed that the problem was also noticeable on some heavier structures (e.g. large road bridges) as early as in the 1950s [4], albeit rarely reported to a wider audience. Some now well-known examples are lateral vibrations of the north side of the Auckland Harbour Road Bridge in New

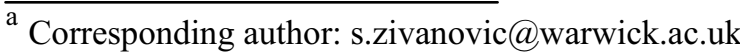


Zealand caused by demonstrations in 1975 [3] as well as a recent sway of the Brooklyn Bridge in New York City which was exposed to an unusual loading scenario of crowd of pedestrians during a power blackout [4]. Another example experienced by the author herself is excessive vibration of the Branko's Bridge in Belgrade triggered by a crowd of protesters during demonstrations in 1990s. In all these cases, strong vibrations were triggered by crowd of pedestrians on structures that did not necessarily appear to be too slender, but simply had at least one vibration mode at a frequency excitable by human activities.
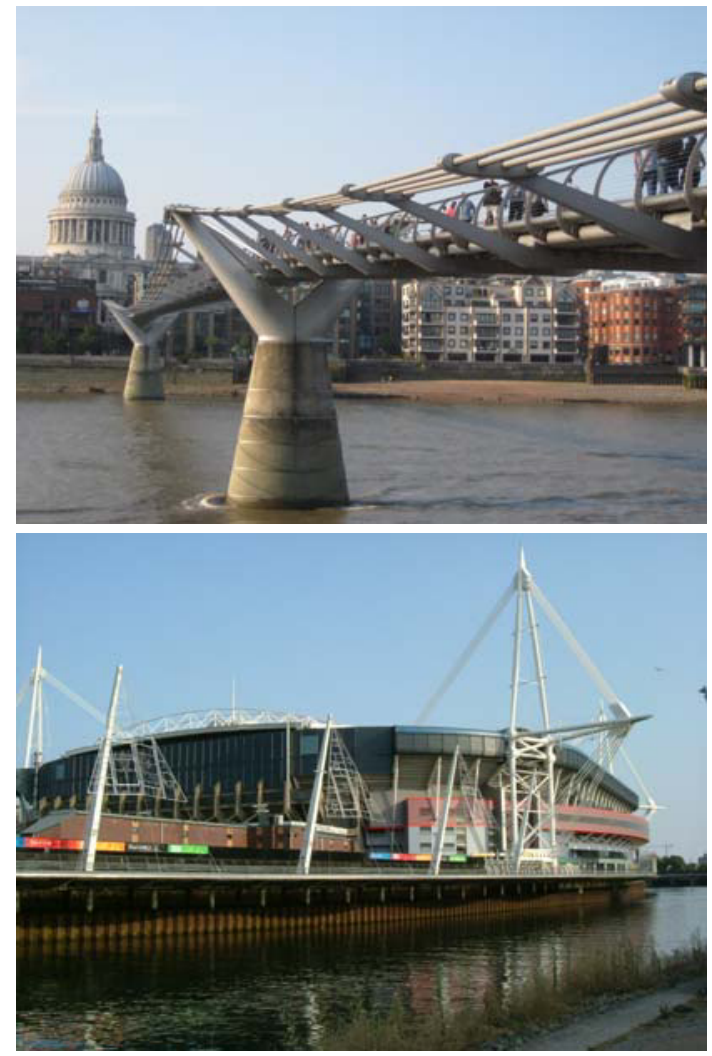

Figure 1. The Millennium Bridge, London and the Millennium Stadium, Cardiff.

It is important to mention that straight forward judgement of the potential of a structure to experience vibration problems cannot necessarily be made by using information on their natural frequency and damping only. Namely structures having similar spans, natural frequencies and damping ratios, do not necessarily behave in the same way dynamically. For example, a bridge made of a novel fibre-reinforced polymer (FRP) composite material with main span of $63 \mathrm{~m}$, natural frequency of $1.5 \mathrm{~Hz}$ and damping ratio of $0.4 \%$ could exhibit vibrations that are an order of magnitude larger than those measured on a steel-box girder spanning $78 \mathrm{~m}$, having natural frequency of $2.0 \mathrm{~Hz}$ and damping ratio of $0.3 \%$. The difference is mainly due to much lighter nature of the FRP structure [5].

The quoted examples suggest that low-frequency structures having vibration mode(s) in the frequency range that overlaps with the frequency range of most potent harmonics of the human induced forces (approximately up to $10 \mathrm{~Hz}$ ) are potentially (but not necessarily) vulnerable to human-induced loading, and among these, the vulnerability increases as the modal mass decreases.

Similar to sharp progress in earthquake and wind engineering that occurred after major disasters caused by these naturally occurring phenomena, it is not surprising that the highly publicised vibration serviceability problems mentioned above led to intensive research in the new millennium. While primary motivation for improved design in wind and earthquake engineering was to save lives, preventing excessive vibration is mainly about improving serviceability of the structure (either through providing required comfort for human users or through satisfying stringent vibration requirements for functioning of vibrations sensitive equipment, such as electron microscopes and equipment for high-precision fabrication found in the semi-conductor facilities). Increased structural sensitivity to vibration requires a better understanding of the nature of the human load and (similar to developments in wind and earthquake engineering) accommodating stochastic nature of the human loading represented by the simple fact that there is a large variability in the human population with respect to the type and duration of action performed, and ground reaction force (GRF) generated in the process.

Of many actions performed by humans on structures (e.g. walking, running, jumping, bobbing, swaying, dancing, etc.), walking, jumping and bobbing are perhaps most relevant for structural engineers, and they are exclusively considered in this paper. Walking represents one of the most natural and most frequently performed activities, relevant for design of building floors and footbridges. Jumping ("launching one's self in the vertical direction, removing the entire body from contact with the ground" [6]) and bobbing ("attempting to 'jump' whilst the feet remain in contact with the ground" [6]), on the other hand, are frequently encountered in venues that accommodate sports or music events, such as grandstands and music halls. Jumping action results in the largest force, but bobbing is more frequent. Although all activities result in generating a $3 \mathrm{D}$ dynamic force, it is the vertical component of the forces studied, as well as the horizontal lateral component of the walking force, that are considered within this paper.

Examples of a vertical and a lateral component of the force generated within a single walking step and a vertical component of a single jump are shown in Figure 2. While the force waveforms, such as those shown in Figure 2, are qualitatively similar when generated by different individuals performing nominally the same activity, it has been acknowledged over the past 15 years that inherent differences between human beings demand utilising a stochastic approach for quantitative description of human actions. Basic principles of this approach have already been embedded in the most recent design guidance for footbridges and floors [2, 7, 8]. However, there are a number of other important questions that remain to be answered. For example, how humans interact with oscillating structures? Guidance for stadia design published by the Institution of Structural Engineers in the UK seven years ago [9] is the only 
guidance available, and it is relevant for bobbing activity only. Furthermore, how people interact with other people, especially in crowds that, occasionally, cross footbridges or, more frequently, jump of bob on grandstands? What vibration level influences the two types of interaction and in which way? Does human's perception of vibration change in this process?

Bearing in mind the complexity of the questions posed and, in general, of the modelling of humans, a successful vibration serviceability assessment of a structure will require integration of diverse disciplines, such as structural engineering, psychology and biomechanics. Significant advances in modelling human actions have been made over the last decade or so. This paper sets to describe some of these developments in relation to walking, jumping and bobbing activities. Given significance of verifying the theoretical models, the experimental work is also presented, whenever possible. The focus is on modelling an individual person only, given that understanding a single person's action is a prerequisite for successful modelling of groups and crowds.

The paper starts with a brief reminder of traditional modelling of human-induced force. It then gradually proceeds to explaining how the challenges in accounting for the variability in the dynamic force, human-structure interaction and human-human interaction are being or could be addressed. At the end, the findings are summarised and recommendations for further research made. Due to space limitations, the paper covers main ideas without attempting to list all references of interest. For more information, an interested reader is directed to a number of excellent review papers published over the last decade that address different aspects of this research theme [4, 6, 10-13].

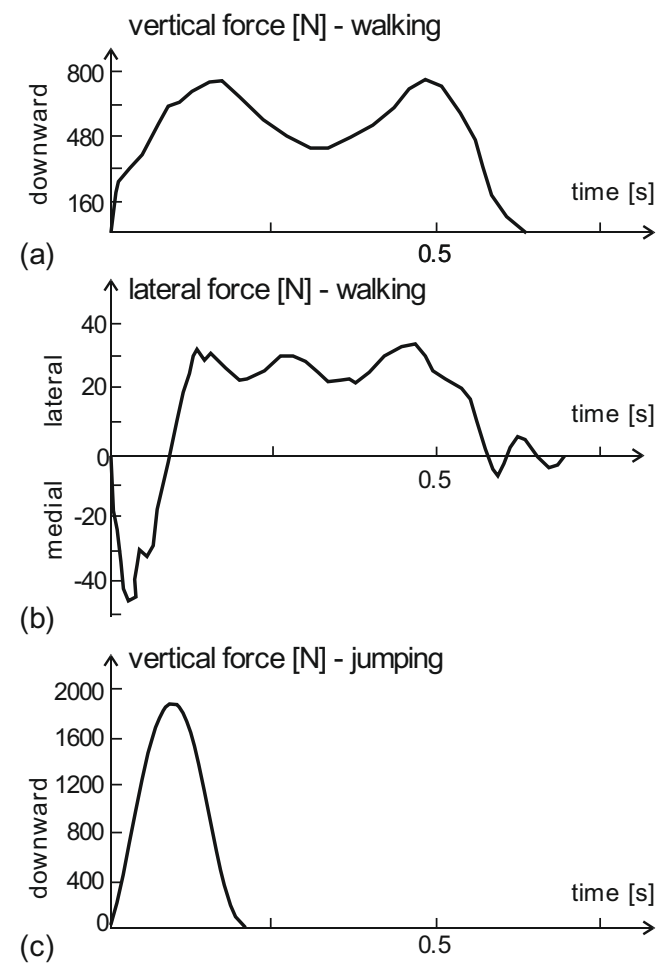

Figure 2. GRF generated while (a, b) walking and (c) jumping.

\section{Traditional modelling}

Until recently, human actions on the structures have been considered by focussing exclusively on the dynamic force generated in the process. Deterministic force models have been employed; the Fourier series representation being most popular due to its simplicity and ability to represent (almost) periodic nature of most human activities. Therefore, the (vertical component of the) ground reaction force $F(t)$ was modelled as a sum of several forcing harmonics:

$$
F(t)=W\left[1+\sum_{i=1}^{N} D L F_{i} \cos \left(2 \pi i f_{a} t+\varphi_{i}\right)\right]
$$

where $W$ is the weight of the person, $f_{\mathrm{a}}$ is the frequency of the activity, $D L F_{\mathrm{i}}$ is the dynamic loading factor for the $i$ th harmonic (i.e. the harmonic amplitude normalised by person's weight), $\varphi_{i}$ is the phase angle for the $i$-th harmonic, and $N$ is the total number of harmonics considered. Frequencies for the walking activity range from $1.4 \mathrm{~Hz}$ to $2.5 \mathrm{~Hz}$, while for jumping and bobbing these are $1.5-3.5 \mathrm{~Hz}$ and $1.5-4.5 \mathrm{~Hz}$, respectively. Given that the first harmonic of the force is usually the strongest, the structures with frequency up to about $5 \mathrm{~Hz}$ are most vulnerable to human actions. However, a few of higher harmonics can also contain sufficient energy to cause excessive vibrations. Figure 3 illustrates relative strength of first four harmonics for walking and jumping actions (vertical direction) and their frequency dependence. While these two features have not necessarily been included in all the relevant design guidelines used in the past, they have been well known by structural engineers for some time and they were normally considered in the structural design. However, the loading case regularly assumed in the vibration serviceability check was that of a person exciting the resonance of the structure by one of the first 4-6 harmonics. While the choice of the worst-case scenario was understandable (and compatible with the philosophy underpinning the ultimate limit state design) 20 years ago, this simplified approach was exposed when more slender structures came into use.
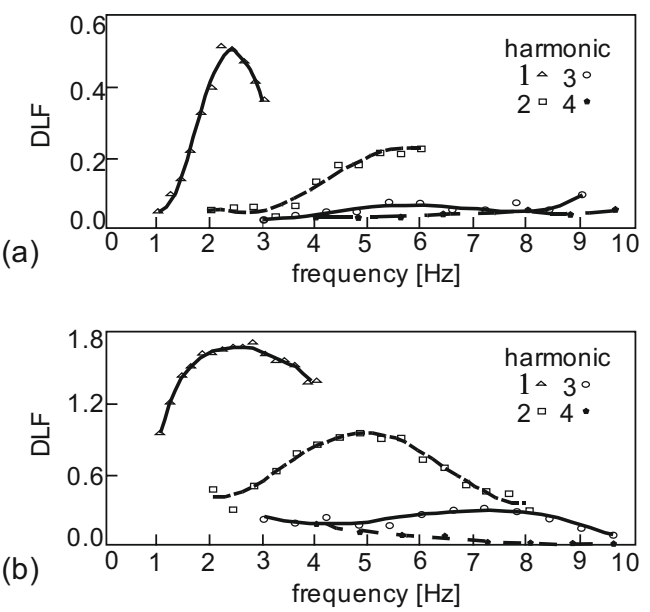

Figure 3. DLF for (a) walking and (b) jumping [14].

Drawbacks of the described modelling gradually came into light after intensified research activities, 
directly or indirectly motivated by the Millennium Bridge problem. These can be summarised in form of four challenges:

- Randomness in the human loading: Inherent diversity in the human population utilising a structure results in a range of activities performed at different frequencies and with different dynamic force output. These inter-subject variations are accompanied by the intra-subject variations. Namely, the same person performing nominally the same activity on different days and in different environments will produce different dynamic outputs. This output by a single person changes even on a cycle-by-cycle basis, as best illustrated by narrow-band spectrum of the GRF [15]. Therefore, it becomes evident that stochastic modelling of human loading is required.

- Human-structure interaction (HSI): Human kinematics, and therefore the resulting GRF, is influenced by vibration of the supporting surface. A crowd of pedestrians interacting with a swaying deck of the Millennium Bridge demonstrated that using models for humans applicable on stationary surfaces is insufficient for successful prediction of structural vibration. This highlighted a need to consider human and structure as two components of a joint human-structure system. Dynamics of this system and its influence on the structural vibration has been investigated intensively over the last 15 years, and a few candidate approaches will be elaborated in this paper.

- Human-human interaction (HHI): People within a group or crowd are influenced by those around them. As a result, the generated GRF might differ from that seen when the person acts on their own. When responsiveness of humans to environmental stimuli, such as music, visual cues or tactile stimuli, is taken into consideration, then the complexity of modelling HHI quickly becomes apparent. This area of research, in structural engineering context, has been mostly focussed on predicting people's potential to synchronise with others, but there exists a need to link it with underlying physiological and psychological factors.

- Human perception and response to vibration: When people, active and intelligent dynamic systems in their own right, are exposed to vibration, they are bound to, consciously or unconsciously, react to it. While the guidelines for human body exposure to vibration do exist and are continuously improving, the question remains how vibration influences human activities and, in turn, the generated GRF? Can this be captured by a generic model of a human or it requires fundamentally different models for different vibration characteristics (such as frequency and magnitude)?

The four challenges have been investigated to various degree and some of them have already been addressed and/or discussed in new design guidelines. The reminder of the paper will present key research in relation to the first three challenges and if/how these are being incorporated in the contemporary design guidance. The fourth challenge is not specifically addressed in this paper apart from some comments made in relation to other topics.

\section{Challenge 1: randomness}

The existence of inter-subject variability in parameters crucial for force definition is now widely known fact and it is usually modelled using Gaussian distribution (for most modelling parameters). In the context of Equation 1, the parameters of interest are DLFs, pacing frequency, body weight and phase lag (usually modelled using a uniform distribution). Furthermore, for walking action, the force is travelling across a structure during limited period of time, and the speed of travel, as well as arrival times (Poisson distribution) are additional parameters to consider. Pedersen and Frier [16] showed that, among four parameters they studied in relation to the walking activity (pacing frequency, speed of travel, DLF and body weight), probabilistic modelling is crucial for pacing frequency only. Inclusions of probability distributions for other parameters are not necessary as long as the population using the structure is described using representative mean values. Perhaps similar conclusions can be drawn for jumping and bobbing actions.

Implementation of randomness of the force into estimation of the vibration response to single person loading in probabilistic sense is straight forward via means of Monte Carlo simulations [17], provided a sufficient number of simulations is performed. Knowledge about sensitivity of the response to randomness in parameters is therefore important for reducing the number of required simulations. Simulating walking activity on floor structures could be especially computationally demanding since apart from including pedestrian arrival time it also needs to accommodate randomness in the walking paths that can be chosen by individuals (interlinking with the demanding subjects of human-human interaction and human's ability to avoid obstacles, such as furniture, in, say, office environments). Further research in this direction would be welcome.

An alternative to Monte Carlo simulations is to utilise random vibration theory [18]. This approach could lead to estimate of probability of exceedance of a particular vibration level over given return period $[19,20]$. The drawback of this approach is that it could only be applied to stationary ergodic processes. Observations on the Podgorica bridge during non-congested pedestrian traffic show that traffic could vary significantly [17]. For example, during a 45 minute-long test conducted during rush-hour traffic, on average 26 people were on the bridge (Figure 4). However, the coefficient of variation in the number of people was quite large at $52 \%$. Despite this, the probability density function representing the vibration response was close to a Gaussian distribution. On the other hand, tests exhibiting less variation in the traffic resulted in more discrepancy between the actual and theoretical (i.e. Gaussian) probability density functions for the vibration response. To achieve a required level of confidence for utilising the random 
vibration theory in the vibration guidelines, there is a need to collect more experimental data over long time intervals so that the hypothesis of the stationarity of the traffic and the corresponding response could be tested.

The probabilistic approach to the vibration assessment has been embedded in some recent design guidelines for floors [8] and footbridges [2, 7]. It has been recognised that the non-exceedance of a pre-defined vibration level should not be absolute, i.e. concentrated on the highest level of possible vibration response which usually has extremely low probability of occurrence. Instead, allowing vibration to exceed a pre-set numerical value with sufficiently low probability of occurrence is adopted. There is no, and perhaps should not be, an absolute consensus about the choice of the sufficiently low probability figure across different structural environments, and different institutions and countries. The floor guide, published by the UK Concrete Society [8], is developed considering the loading that has $25 \%$ probability of exceedance, while the footbridge guides are more stringent: the Sétra guideline chose the probability of non-exceedance of $95 \%$ [2] while the national Annex to Eurocode 1 in the UK [7] specifies a more relaxed level of $89 \%$. This flexibility is a positive development inherent to the probabilistic approach since it allows adopting a parameter that is most suitable for local or national conditions. Therefore, it can be said that the vibration serviceability design has moved away from the design against the worst-case scenario towards a more sophisticated probabilistic approach that is more suitable for addressing the vibration serviceability state.

The second type of randomness (intra-subject variability) is more subtle than the inter-subject variability commented above. This type of randomness in the loading is relevant for development and calibration of high-fidelity models of humans, and in addition, it could be crucial for accurate prediction of the vibration response $[15,21]$. An example of the intra-subject variability is variation in the pacing frequency at a particular walking speed. Figure 5a shows the coefficient of variation $(\mathrm{CoV})$ measured on a population of ten pedestrians. Similarly, the variation in the frequency measured on a population of eight TSs during jumping and bobbing is shown in Figures $5 \mathrm{~b}$ and $5 \mathrm{c}$, respectively. It is apparent from Figure 5 that the activity frequency is most consistent in case of walking, followed by jumping, and then bobbing. Other parameters, such as step length and width (walking), or peak force and contact duration (jumping) can be presented in a similar way.

Continuous improvements in human-loading modelling will eventually result in high-fidelity models capable of accounting for all these uncertainties. In this process, care should be taken that some parameters are mutually dependent. For example, while peak force and contact ratio exhibit jump-by-jump variations, they are also correlated as shown in Figure 6. There is currently no simple guidance aimed at the structural engineers about incorporating this type of relationship in the probabilistic models; however there are a number of modelling proposals available in literature concerned with both walking [20, 24-28] and jumping/bobbing activities $[29,30]$.

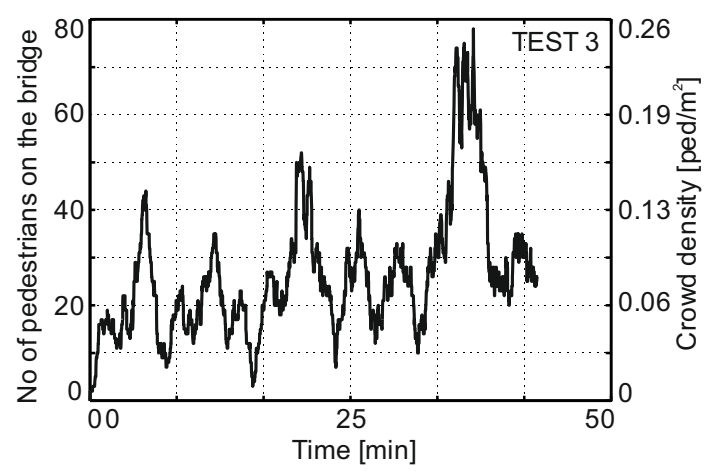

Figure 4. Traffic fluctuations on the Podgorica bridge [17].
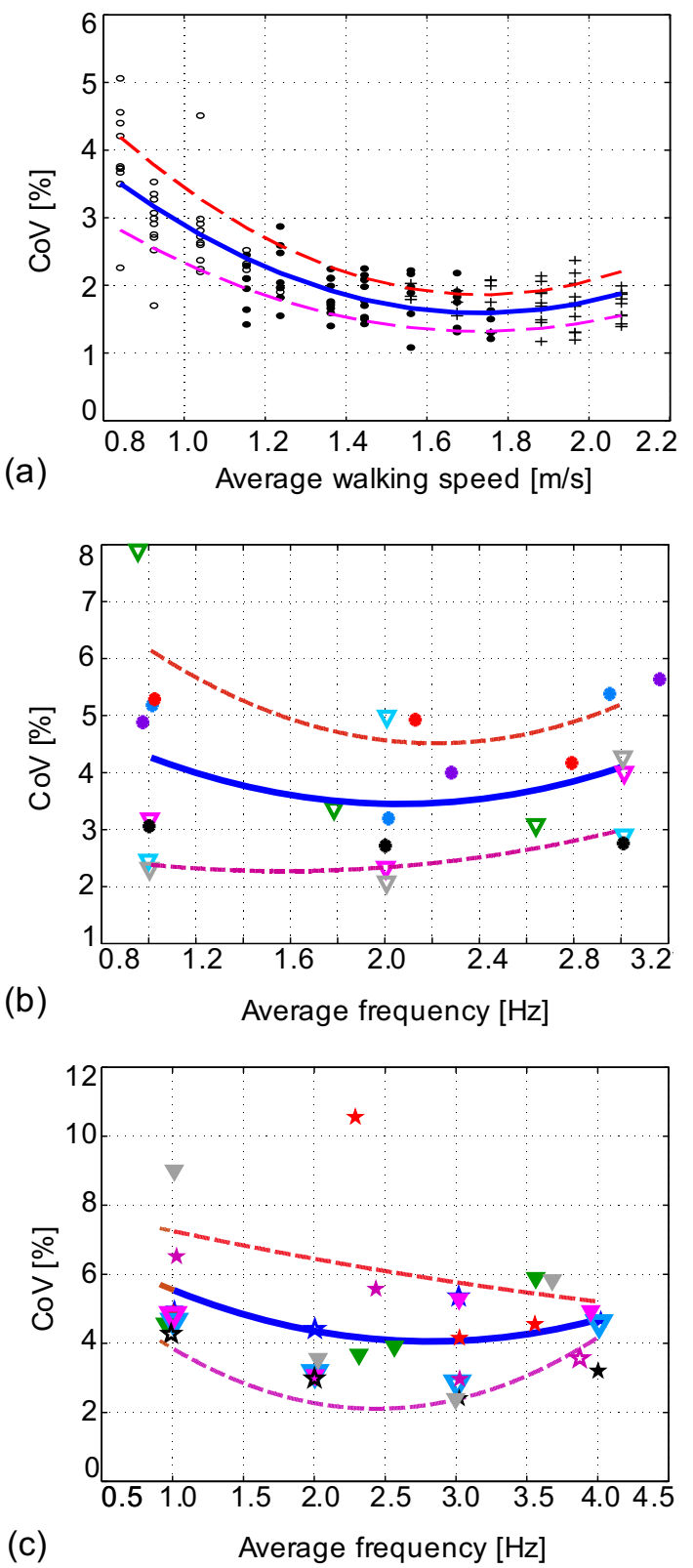

Figure 5. CoV for pacing rate while: (a) walking [22] and (b) jumping and (c) bobbing [23].

Detailed knowledge of parameters characterising human activities is valuable, especially for better understanding of human locomotion and dynamics of the 
human body. To which extent detailed modelling is to be included in the design practice will depend on finding the balance between the need for such a detailed modelling and the computing efficiency.

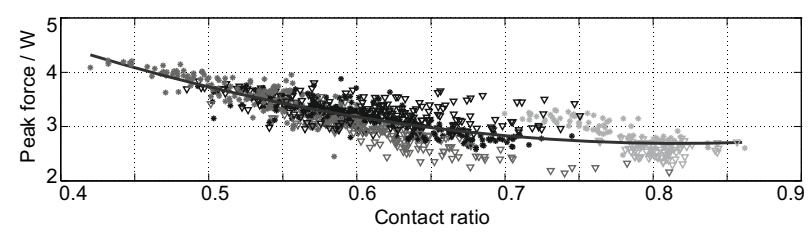

Figure 6. Peak force as a function of contact ratio for jumping activity [23].

\section{Challenge 2: HSI}

Humans are inherently unstable, since they are of bipedal nature and two thirds of their mass is located at two-thirds of their height [31]. Exposure to and interaction with oscillating supporting surface could further compromise human stability and influence the force induced into the structure. This challenge is perhaps most researched among the four, as a direct legacy of the Millennium Bridge problem. A number of different approaches have emerged and they will be presented in this section in order of their entrance into the structural engineering sector.

\subsection{The Millennium Bridge legacy}

Excessive lateral accelerations of $2.0-2.5 \mathrm{~m} / \mathrm{s}^{2}$, corresponding to lateral displacement amplitude of up to $70 \mathrm{~mm}$, occurred on the London Millennium Bridge on its opening day in June 2000. A dense crowd of pedestrians crossing the bridge caused these unexpected vibrations. At the same time, no excessive vertical vibration was recorded [3].

The event was reported in numerous press articles and broadcasts around the world, initiating new lines of research into vibration serviceability. The bridge was closed, and Arup, the engineers, conducted internal research of the problem. Since the excessive sway could not be predicted by pedestrian modelling known at the time, the focus was on unearthing the cause of sudden vibration increase seen on the bridge both on the opening day and during experimental investigations that followed [3]. The conclusion made by Dallard et al. [3] was that pedestrians, on average, act as negative dampers when exposed to the lateral vibrations, and produce a force that is proportional to the velocity of the bridge (Figure 7). The onset of the rapid vibration increase was explained as a point at which people's negative damping overcame the positive damping of the bridge. This model has shown some promise to predict vibrations on other structures. However, its assumption that all pedestrians make equal contribution to the vibration response is clearly a simplification that neglects the inter-subject variability in the pacing and other parameters. Soon after Arup's research findings were reported, a refined modelling of a pedestrian became the focus of worldwide research, some of which is reported in the next three sections. An additional question that emerged was whether people in crowds synchronise with the structure, or between themselves, or both, and under which conditions this occurs.

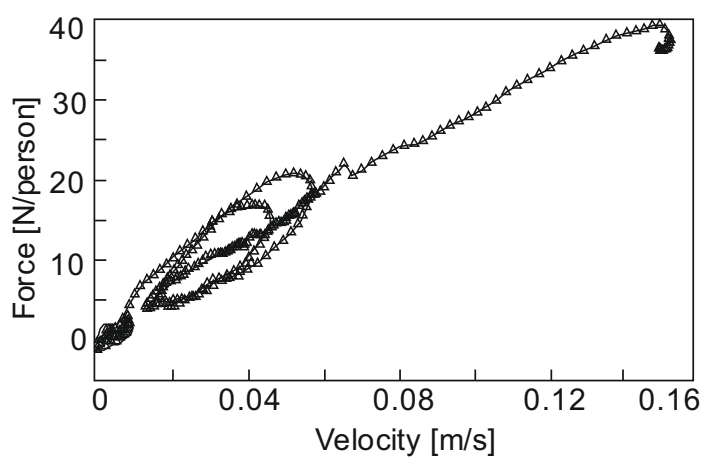

Figure 7. Lateral force per person vs deck velocity [3].

\subsection{Human body as MCK model}

Human body is a complex dynamic system that has its own mass, stiffness and damping [32]. Dynamics of passive, e.g. standing or sitting, humans is often successfully modelled as a mass-damper-spring (MCK) SDOF system. After an extensive literature review, Jones et al. [6] found that reported values of a damping ratio range from $33 \%$ to $69 \%$, the natural frequency from $3.3 \mathrm{~Hz}$ to $10.4 \mathrm{~Hz}$, while the mass is close to the physical mass of the body. It is only natural to investigate applicability of this simple approach to modelling of more challenging active postures.

Identification of the dynamic properties for an active person is a difficult task as these properties are affected by continuous changes in the body posture and alternations in tensing and relaxing various muscle groups during movements [6]. Similar to modelling passive people, a possible way forward is to identify an average damping, frequency and stiffness that fit some sort of the observed experimental data.

The MCK model, crossing a bridge at a constant speed together with a harmonic force induced into the structure, was first proposed for modelling people walking by Archbold et al. [33]. The harmonic force represents the force that is normally induced when walking on stationary (non-oscillating) surfaces while the role of the MCK oscillator is to modify the force to account for the HSI. The latter is achieved through accounting for the damping and spring forces generated due to relative motion between the mass of the human oscillator and the mass of the structure. Acrhbold et al. carried out the response simulations on a virtual structure for varying properties of the human oscillator. They showed that the use of the MCK model can significantly alter the response that would otherwise be generated under the harmonic force alone. The difference was most significant when pacing frequency was close to the natural frequency of the structure. The authors acknowledged that the calibration of the model and its verification on as-built structures need to be addressed if the model is to be implemented in the design practice. 
Help perhaps could be sought from the research carried out in the field of biomechanics of human gait. Stiffness and damping of lower body parts are often investigated as they provide useful information for diagnostics in medical and sports applications and design of prosthetics and artificial limbs. For example, Geyer et al. [34] reported leg stiffness in the range $5-50 \mathrm{kN} / \mathrm{m}$, with higher values typical of faster walking speeds at which the leg muscles seem to be tenser and less deformable. Kim and Park [35] found that the damping ratio for a leg ranges between $2.2 \%$ and $7.0 \%$ while the stiffness normalised by the weight to height ratio is between 45 and 75 . For a person having mass of $75 \mathrm{~kg}$ and being $1.8 \mathrm{~m}$ tall, the corresponding stiffness is in the range 18$30 \mathrm{kN} / \mathrm{m}$, while the corresponding damping coefficient is between 50 and $210 \mathrm{~N} /(\mathrm{m} / \mathrm{s})$. Note that the findings from these studies represent dynamic properties of a leg featuring bipedal models commonly used in biomechanics, and therefore are not necessarily directly applicable to calibration of the MCK oscillator. However, the presented figures serve as a good indicator of the expected order of magnitude for the parameters of interest.

With the aim to identify MCK properties in the walking posture, the author of this paper performed simulations aimed at matching the measured peak response of the FRP bridge mentioned in Section 1. Data from nine tests performed by six test subjects, crossing the bridge one at a time, were utilised. The identified natural frequency of the walking human was from $1.0 \mathrm{~Hz}$ to $2.6 \mathrm{~Hz}$, while the damping ratio was mainly in the 10 $27 \%$ range. Using the actual mass of the test participants, this resulted in the body stiffness between $4 \mathrm{kN} / \mathrm{m}$ and $22 \mathrm{kN} / \mathrm{m}$. Interestingly, the identified stiffness is comparable with the values reported for bipedal models, while damping ratio seems to be larger.

An example of the measured time-domain response when walking to excite resonance is shown in Figure 8a, alongside an envelope of the simulated response (solid lines). In addition, an envelope presenting the response obtained when only harmonic force is used is shown as dashed lines. It can be noticed that ignoring the HSI leads to the peak structural response of about $3.3 \mathrm{~m} / \mathrm{s}^{2}$, overestimating the measured peak 2.7 times. When the interaction model is utilised in the simulation not only that the peak response matched the measured response, which is expected given that the pedestrian properties used in the simulation were calibrated against the measured peak in the first place, but more interestingly the shape of this response envelope is much more similar to that measured. Another example of a measured response acquired for out-of-resonance walking and the corresponding envelope of the simulation response are shown in Figure 8b. Again, the shape of the simulation response time history is reasonably comparable with that measured.

The system of equations that represents the $2 \mathrm{DOF}$ model composed of MCK and harmonic force moving over a SDOF (single mode) model of the bridge is:

$$
\begin{aligned}
& m_{p} \ddot{y}_{p}(t)+c_{p}\left(\dot{y}_{p}(t)-\dot{y}_{b}(t) \Phi(x(t))\right)+ \\
& k_{p}\left(y_{p}(t)-y_{b}(t) \Phi(x(t))\right)=0
\end{aligned}
$$

$$
\begin{aligned}
& m_{b} \ddot{y}_{b}(t)+\left(c_{b}+c_{p} \Phi^{2}(x(t))\right) \dot{y}_{b}(t)-c_{p} \Phi(x(t)) \dot{y}_{p}(t)+ \\
& \left(k_{b}+k_{p} \Phi^{2}(x(t))\right) y_{b}(t)-k_{p} \Phi(x(t)) y_{p}(t)=F(t) \Phi(x(t))
\end{aligned}
$$

where $m, c$ and $k$ denote mass, damping and stiffness for the pedestrian (subscripts $p$ ) and the bridge (subscript $b$ ) DOF. Similarly, $y$ and its derivatives represent displacement (and velocity and acceleration) of the bridge and pedestrian DOF, while $\Phi$ denotes the mode shape.
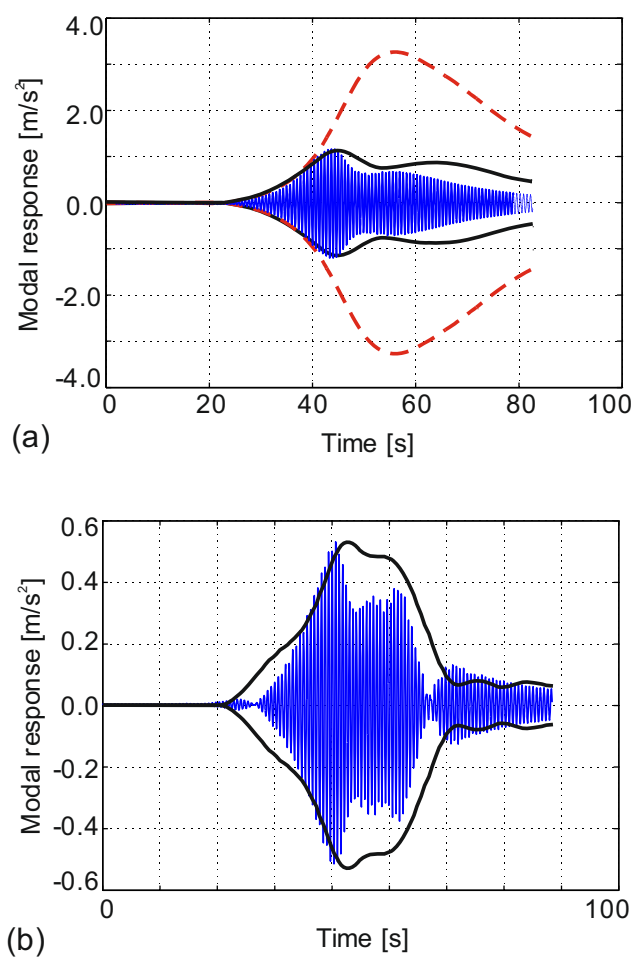

Figure 8. Measured modal responses (fully shown) and MCK simulation response envelopes (solid lines) for walking at a pacing frequency in (a) resonance and (b) out-of-resonance with the bridge. Dashed line in (a) presents the response envelope under moving force only.

Solving an eigenvalue problem at each instant of time leads to identification of eigenvalues and eigenvectors, from which the natural frequencies and modal damping ratios of vibration modes can be calculated [36]. Figure 9 shows temporal development of the natural frequencies for a 2 DOF system while a pedestrian is crossing a virtual $50 \mathrm{~m}$ long footbridge having half since mode shape. The footbridge frequency is set to $1.5 \mathrm{~Hz}$. Three values of pedestrian frequency were considered: $1.37 \mathrm{~Hz}, 1.5 \mathrm{~Hz}$ and $1.62 \mathrm{~Hz}$. The lines of the same type in Figure 9 show evolution of natural frequencies of both the pedestrian $(p)$ and the bridge $(b)$ in each of the three simulations. It can be noticed that the two natural frequencies always move away from each other. In addition the largest change in any of the two natural frequencies (about 4.4\%) occurs when the starting pedestrian and bridge frequencies are equal. The largest deviation from the initial frequency for each DOF is shown in brackets in Figure 9. These maximum changes of natural frequencies are those that would be obtained for a $2 \mathrm{DOF}$ system in which the moving pedestrian is replaced by a stationary ("vibration absorber") pedestrian "installed" in the midspan. This evolution of the natural frequencies of the 2DOF system 
with time is behind the ability of the model to simulate the response in the "resonance" condition seen in Figure 8 a.

The ability to simulate satisfactorily the shape of the measured response envelope suggests that the model might have potential to capture the interplay of the dynamics of the two system components. However, to make a confident conclusion requires further experiments in which all relevant parameters (especially the pacing frequency and the GRF) would be better controlled and more reliably measured than it was the case in the current set of experiments (that were designed to investigate some other effects of the bridge response not related to the current study). There is also a need to experimentally check whether the human body parameters are a function of the vibration level and frequency. It is difficult to expect that MCK values for the human body remain vibration independent. Should this be confirmed then the model would need to be calibrated across a range of different testing conditions.

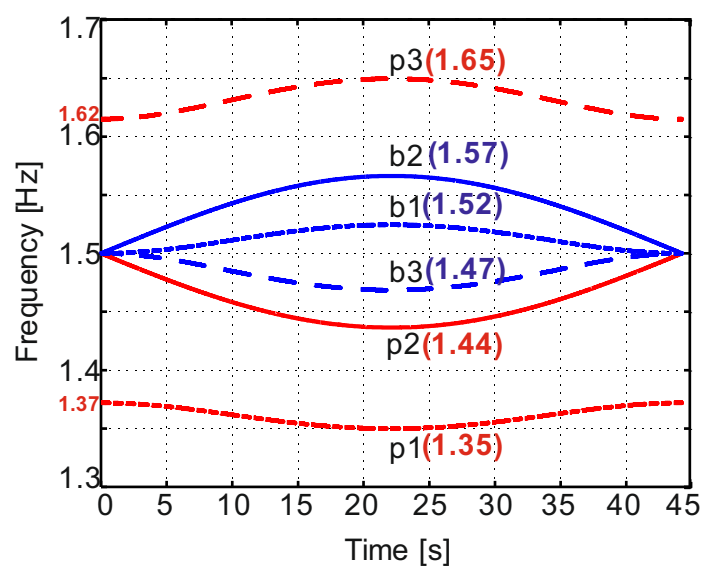

Figure 9. Temporal development of natural frequencies of three 2DOF systems. $b$ - bridge DOF, $p$ - pedestrian DOF. The values in brackets (in $\mathrm{Hz}$ ) show largest deviations of the frequency from its initial value for both DOFs in each of the three simulations.

\subsection{Human body as AMCK model}

In addition to the MCK system, the human body in the AMCK model is represented by an internal (instead of external) harmonic driving force (i.e. actuator, A) acting against both the pedestrian mass and the structure DOF (Figure 10). The GRF induced into the structure is equal to the sum of the actuator force, and the stiffness and damping forces of the oscillator. This model has not been implemented for pedestrian modelling yet. Instead it has been proposed for (and adopted in) modelling bobbing actions on grandstands [37]. To identify the damping, stiffness and the internal forces, Dougill et al. optimised these parameters so that the output force matched that measured on the rigid surface. As a result, they reported an average damping ratio of $25 \%$ and natural frequency of $2.3 \mathrm{~Hz}$, while the oscillator mass was assumed equal to the physical mass of the person's body [9, 37]. For a person having mass of $75 \mathrm{~kg}$, the equivalent stiffness is about $16 \mathrm{kN} / \mathrm{m}$, the corresponding damping coefficient is $540 \mathrm{~N} /(\mathrm{m} / \mathrm{s})$ while the amplitude of the first harmonic of the actuator force is $20-30 \%$ of the body weight. However, the authors noticed that their model having constant and vibration independent damping, stiffness and actuator force cannot genuinely represent the contact force on very lively structures. A likely reason is that the contact force and consequently the parameters of the AMCK model depend on the vibration amplitude and frequency, and therefore separate calibration of the model is required for different vibration properties, similar to the conclusion made for the previous model.

What is important to notice in relation to this model is that it is a rare model for HSI currently implemented in the design guidance [9]. Therefore, it is reasonable to expect that, due to use of the model by the structural engineers, a critical evaluation of the performance of the model will be possible in near future.

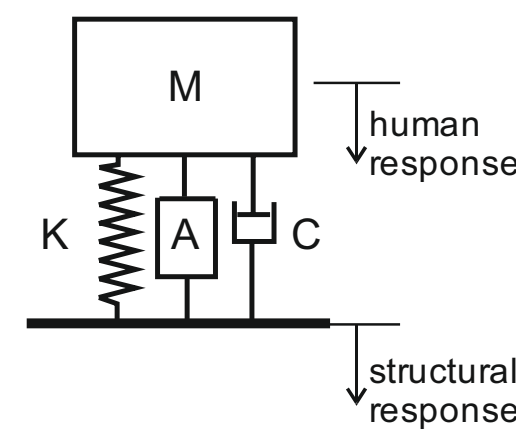

Figure 10. AMCK model of a human.

\subsection{Human body as IPM}

When it comes to modelling walkers, the bi-pedal nature of walking locomotion is often a motivation behind employing inverted pendulum models (IPMs). This type of modelling has traditionally been used in the biomechanics research and it includes a variety of models, as shown in Figure 11 [38]. Structural engineers started employing the IPM model initially to explain lateral sway on the Millennium Bridge [44]. Since then, the model has also been utilised for modelling walkers exposed to the vertical vibration.
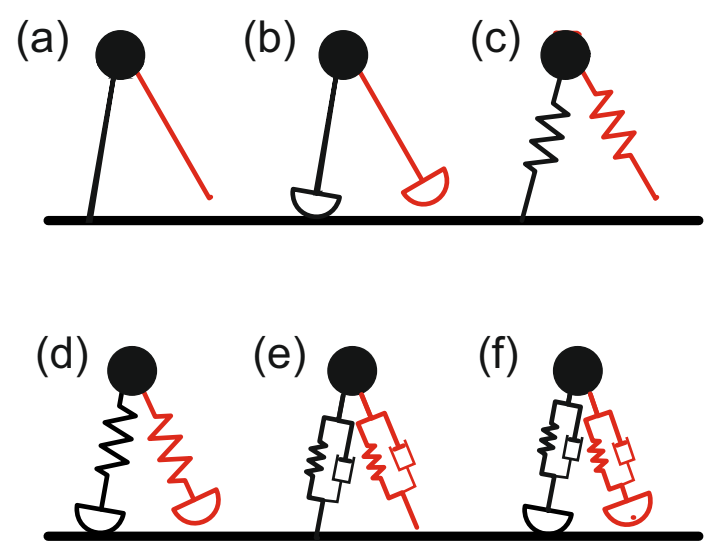

Figure 11. Bipedal walking locomotion models: (a) inverted pendulum [39], (b) rocker foot [40], (c) spring mass [41], (d) spring mass with rocker foot [42], (e) spring mass with damper [43] and (f) spring mass with rocker foot and damper [35]. 


\subsubsection{Lateral vibration}

Use of bipedal models in structural engineering research started with Barker's idea to utilise the simplest IPM (see Figure 11a) to study interaction of pedestrians with swaying bridges. He resolved the static (body weight) force into a lateral (i.e. frontal plane) component by using the information of the leg inclination angle [44]. He assumed that the body centre of mass (BCoM), represented in the model by the point mass, travels forwards in a straight line, uninfluenced by the bridge sway. The lateral force calculated in this way is a function of the inclination angle that is influenced by the deck position. The achieved lateral force evolution within successive strides led to the conclusion that the pedestrians do the work on the bridge regardless of their pacing frequency. This remarkably simple model managed to simulate the lateral instability noticed on the Millennium Bridge without enforcing pedestrians' synchronisation with the bridge, which has been a most frequent "theory" mentioned in the context of the bridge's excessive sway. However, the model required further refinement to account for an actual, rather than a straight line, movement of the BCoM, and to introduce better representation of the foot placement in each step.

Macdonald [45] achieved the two improvements by utilising a model of human balance developed in the research field of biomechanics [46]. As in the case of Barker's model, he assumed instantaneous transfer of body from one foot to another (basically neglecting the double support phase in the walking cycle), but implemented a more realistic foot placement strategy based on the final displacement and velocity of the $\mathrm{BCoM}$ from the preceding step. The lateral acceleration of the BCoM $\ddot{w}$ in his model (Figure 12) is as follows:

$$
\ddot{w}=-\ddot{x}_{b}-\Omega_{p}^{2}(u-w)
$$

where $\ddot{x}_{b}$ represents the lateral acceleration of the structure, $u-w$ is the relative displacement between the centre of pressure for the supporting leg and the BCoM, $L$ is the pendulum length and $\Omega_{p}=\sqrt{g / L}$.

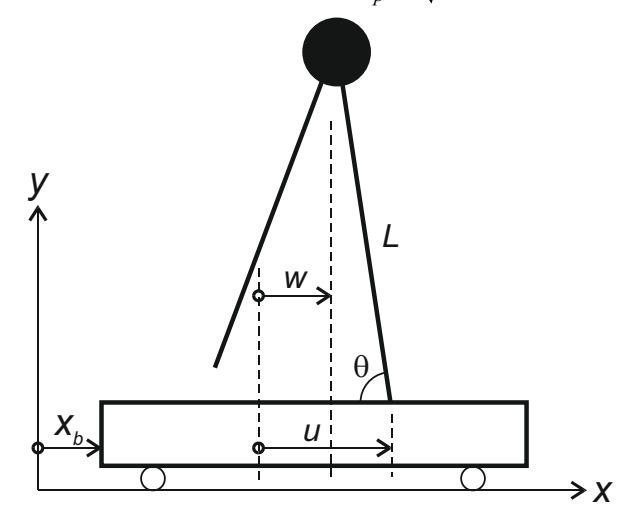

Figure 12. Pedestrian as an IPM on a swaying deck.

Foot placement in each step, required to achieve a stable gait, is taken as:

$$
u=w_{0}+\frac{\dot{w}_{0}}{\Omega_{p}} \pm b_{\min }
$$

where $w_{0}$ and $\dot{w}_{0}$ are the initial displacement and velocity for the BCoM in the observed step, while $b_{\min }$ is the stability margin which is taken as positive value for the right and negative value for the left foot.

Macdonald's modelling provided first insight into differences between a force generated by a pedestrian on a swaying bridge deck and that induced on a stationary (i.e. non-moving) surface. While the main odd harmonic components appeared in the force spectrum in both cases (three dominant harmonic components in Figure 13), the walking on the oscillating bridge generated additional harmonic components at frequencies at the distance of $f_{\mathrm{b}}$ $f_{p}$ either side of the main harmonics (thick, sideway harmonic lines in Figure 13). Among these, the harmonic that matches the natural frequency of the bridge is of most interest. This motion-induced component is known as a "self-excited force" and it is an important consequence of the interaction between the pedestrian and the swaying bridge.

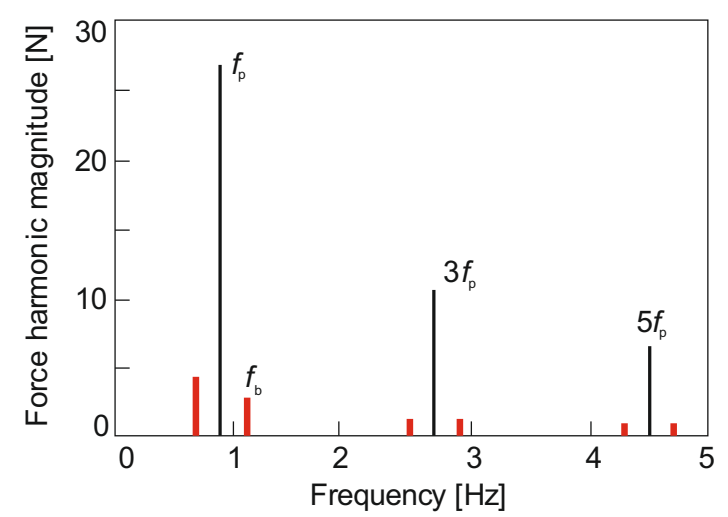

Figure 13. Amplitude of the Fourier spectrum of the lateral force induced by a pedestrian generating the force having fundamental frequency of $f_{\mathrm{p}}=0.9 \mathrm{~Hz}$ on a bridge oscillating laterally at frequency $f_{\mathrm{b}}=1.11 \mathrm{~Hz}[45]$.

The experimental evidence of the existence of the self-excited force was initially provided by Pizzimenti and Ricciardelli [47] by measuring the lateral force induced by a walker on a swaying treadmill. This was furthered in a set of similar, but more comprehensive, experiments by Ingólfsson et al. [48]. The experiments involved 71 test subject and they were performed at swaying frequencies between $0.33 \mathrm{~Hz}$ and $1.07 \mathrm{~Hz}$ and with amplitudes between $4.5 \mathrm{~mm}$ and $48 \mathrm{~mm}$. The selfexcited force was split into components in phase with velocity and acceleration of the structure, and then expressed in the form of additional damping and mass. It was confirmed that pedestrians consistently input the energy into the oscillating structure. The damping component was found to be a function of vibration amplitude. Interestingly, increase in the vibration amplitude led to a decrease in negative damping, implying that the there is a physical limit to the vibration instability development. As for the mass parameter, the pedestrians were observed to add mass to the system when walking at higher frequencies, while the opposite occurs for lower frequencies. Overall, the behaviour was 
also amplitude dependent, adding complexity to the interaction phenomenon.

In the next step, Carroll et al. [49] looked in more detail at the mechanisms by which pedestrians balance themselves on a swaying platform. They identified two strategies that could have adverse effect on the structure. Namely, when pedestrian's forcing frequency does not match the frequency of the structure, the gait width modulation (in itself a consequence of the foot placement strategy) results in the (previously described) motion induced force at the frequency of the structure that resonates with the structural motion. On the other hand, when pedestrian's forcing frequency is synchronised with that of the structure, the person widens the step, which in turn leads to an increase in the GRF.

\subsubsection{Vertical vibration}

Inspired by progress of the modelling of pedestrians in the frontal plane, Bocian et al. [50] utilised the simplest IPM model to perform a theoretical study into the effects of the vertical vibration on pedestrian locomotion in the sagittal plane. They concluded that the GRF, once again, consists of the harmonic components routinely seen when walking over stationary surfaces as well as the motion-induced self-excited force. This force was converted into the equivalent mass and damping, to discover its potential to both add and subtract damping and mass to the structure. However, differently from the lateral direction, it was argued that the most likely effect of the interaction is a beneficial reduction in the structural vibration response. This observation is in line with qualitative observations from the field tests that pedestrians act as positive dampers when the vertical vibration is concerned [21, 51-54].

Dang [38] set to test how a vertically vibrating deck of the laboratory bridge at the University of Warwick (Figure 14) influences the pedestrian locomotion in more detail. He found that vibration of the bridge increases the intra-subject variability in most gait parameters, including the pacing frequency and confirmed the existence of the self-excited force component. This force was found to increase with an increase in the vibration level, as illustrated in Figure 15. Interestingly, for walking at the pacing frequency that matches the vibration frequency of the structure (not graphically presented in this paper), the self-excited force mainly combined with the non-stationary surface force in such a way to reduce the resulting vibration effects. This finding is in contrast with that observed on the laterally moving platform [49], and it makes adverse effects due to HSI less likely on the vertically oscillating structures.

Another interesting finding from Dang's study is that pedestrians are less sensitive to vibrations when walking at higher pacing rates. This is intuitively expected since the characteristics of fast walking (less time spent in continuous contact of a foot with the vibrating surface, and higher body acceleration when walking fast) contribute to reduced sensitivity to deck vibration.

Dang's study involved thee test subjects and three vibration levels, and it would be of benefit to expand it on larger number of test participants and a wider range of test conditions.

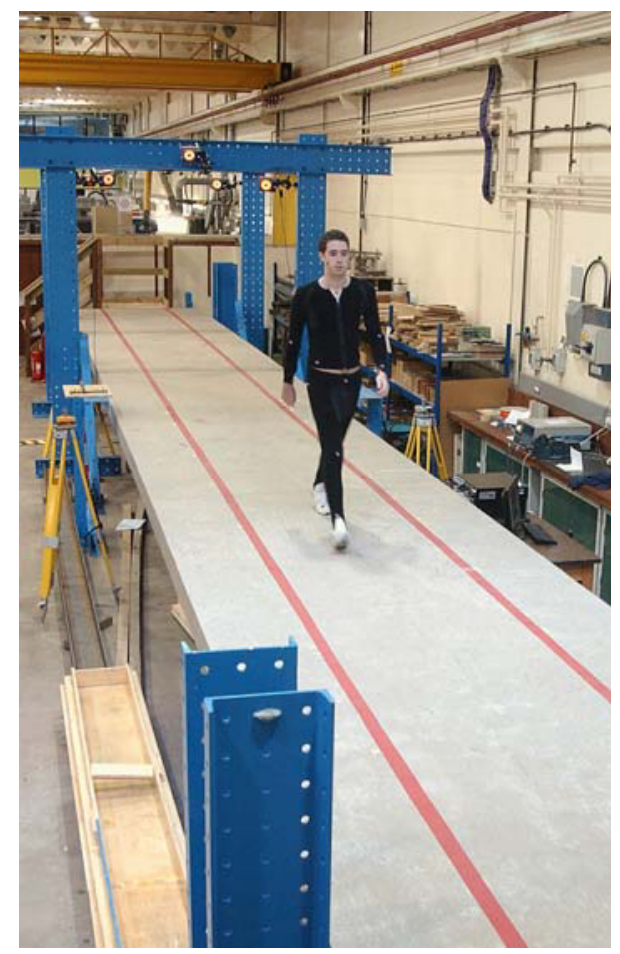

Figure 14. A walking test on the Warwick Bridge.

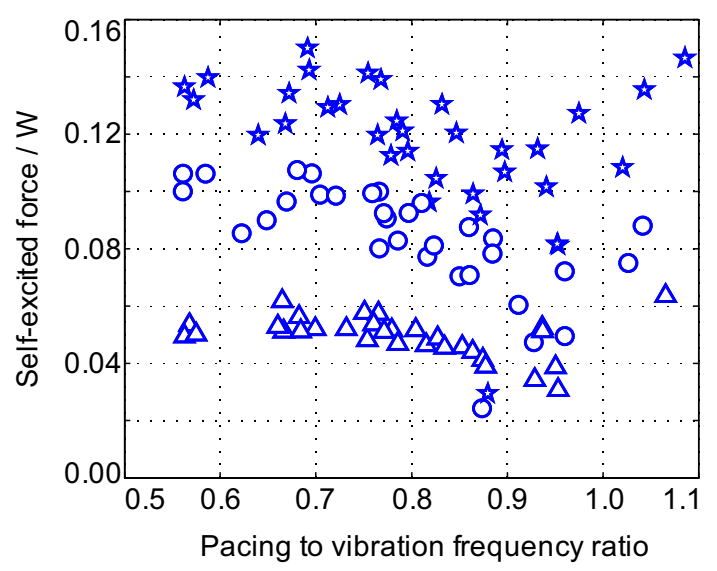

Figure 15. Self-excited force normalised by pedestrian weight as a function of the pacing to vibration frequency ratio. Data are presented for different level of pre-induced steady-state acceleration of the bridge structure: triangles: $0.5 \mathrm{~m} / \mathrm{s}^{2}$, circles: $0.85 \mathrm{~m} / \mathrm{s}^{2}$, stars: $1.2 \mathrm{~m} / \mathrm{s}^{2}$.

\section{Challenge 3: HHI}

Pedestrians walking over footbridges or in offices, spectators in grandstands watching sports events, concert goers in music halls, people attending fitness classes in gyms - in all these situations people performing a dynamic action (and therefore generating a GRF) are surrounded by other people. Regardless of the other participants being friends or complete strangers, a person in any one event is likely to interact with other individuals. This interaction could be complex and is 
usually influenced by both physiological and psychological factors [55].

In those environments when people are likely to attempt to jump, bounce or dance in unison, structural designers are most concerned with ability of people to synchronise one with another, i.e. perform an activity at the same frequency and with little or no phase lag. This becomes a concern when the frequency of the crowd's action coincides with a structural natural frequency. Therefore, the ultimate aim in the design is to ensure that high level synchronisation will not be achieved for example by ensuring that structural natural frequency is outside the vulnerable range [9], or by controlling crowd behaviour. If the crowd is composed of pedestrians on, say, footbridges then the influencing factors might change during a single crossing due to change of spatial position of the person observed, as well as those in their vicinity. Visual, tactile, aural and other stimuli all play the role in both types of settings, and they are widely researched [23]. This section will however exclusively concentrate on a brief discussion of the experiences from social science research into pedestrian dynamics that, so far, was less visible to the structural engineers.

Modelling pedestrian flows (or pedestrian dynamics) has been intensively studied in the context of safe use of critical infrastructure (such as airports, public transport stations and urban spaces) and efficient crowd management. In computational social science, the interaction between individuals is empirically observed and numerically modelled and used for understanding emerging collective behaviour [56]. In this way, complex relationships in the pedestrian flows at bottlenecks, or their special and temporal fluidity could be characterised. The lessons learnt can be implemented in say shaping of walkways in order to prevent or mitigate against potentially high levels of structural vibration [57].

A way to observe emerging crowd behaviour based on microscopic model of pedestrians is to utilise discrete element theory [20]. This theory makes use of individual characteristics of pedestrians as well as empirically observed behavioural rules to simulate movement of pedestrians in their natural environments. The factors of interest are, for example, the motivation of pedestrian to reach the intended location in planned time, and need to avoid collisions with other pedestrians and physical obstacles encountered en route. Carroll et al. [20] proposed integrating this model into simulations of the pedestrian traffic for evaluation of the vibration serviceability state of the structure and pointed out that this approach could be a powerful and cost effective tool for the design of new structures and implementation of the traffic calming measures (e.g. temporary potted threes) during exceptional, rarely occurring, events. Successful implementation of this modelling approach requires good understanding of pedestrian behaviour in the vibrating environment, including conditions that might lead to pedestrian's step synchronisation with their neighbours or with the structure itself. Therefore, to incorporate the pedestrian flow models available in social science research requires to successfully address the second (HSI modelling) challenge presented in this paper.

\section{Conclusions and recommendations for further work}

This paper describes a rapid development of pedestrian modelling strategies in the structural engineering context since the start of the new millennium, also illustrated in extensive literature reviews on the subject $[4,6,10-13]$. From modelling a human dynamic force exclusively by means of the Fourier harmonic components, the modelling has since diversified to accommodate randomness in the dynamic force and include humanstructure integration to the point that some of these approaches are incorporated in the contemporary design practice. In addition, considerations are also being given to the human-human interaction, and understanding to which extent this is influenced by interaction with the structure (and vice versa).

The main conclusions and pointers for further research can be summarised as follows:

- Randomness in the human loading: The vibration serviceability design has moved away from the design against the worst-case scenario towards a more sophisticated probabilistic approach that is more suitable for addressing the vibration serviceability state. This is especially true with respect to accurate modelling of the inter-subject variability. On the other hand, while the importance in the intra-subject variability is generally recognised, there is a need for developing appropriate design procedures that are realistically transferable to the users in the structural engineering sector.

- Human-structure interaction (HSI): It is recognised that the HSI plays an important role in the design of light and slender structures. Models based on body dynamics have been implemented for both the walking and bobbing activities, and the latter is an integral part of the design guidance [9]. The bipedal walking locomotion is also frequently modelled using inverted pendulum models (IPMs), for modelling HSI in both lateral and vertical directions. Both types of models (IPMs and SDOFtype models of the human body) show ability to simulate at least some aspects of the vibration responses seen in practice. There is now a need to move from qualitative to quantitative assessment of the most recent models. The emphasis should be on defining model parameters that can realistically represent population of structural users and verifying the models on full-scale structures.

- Human-human interaction (HHI): Great lessons can be learnt about people interacting within crowds from research on pedestrian flows from other research fields. These could be adopted for the structural engineering applications, provided new understanding on vibration influence on the human interactions is developed first.

- Human perception and response to vibration: While this topic has not been explicitly addressed in the paper, it is nevertheless interlinked with the first three challenges. For example, recent work [38] 
demonstrated that increasing (vertical) vibration level leads to an increase in variations of parameters characterising successive walking steps. At which vibration level these changes become significant? Can they be simulated by the HSI models currently being proposed? Can a single model be used for human exposure to a range of vibration conditions? How does vibration influence HHI? These are some questions expected to be addressed by the research community over years to come, leading to a truly integrated modelling of humans on lightweight, slender structures.

\section{Acknowledgements}

The author would like to thank to the UK Engineering and Physical Sciences Research Council who supported some research described in this paper [grant number EP/I03839X/1: Pedestrian interaction with lively lowfrequency structures, and grant number EP/M021505/1: Characterising dynamic performance of fibre reinforced polymer structures for resilience and sustainability].

\section{References}

1. British Standards Institution, Steel, concrete and composite bridges-part 2: specification for loads; appendix $C$ : vibration serviceability requirements for foot and cycle track bridges, BS 5400. London (1978)

2. Sétra, Footbridges: assessment of vibrational behaviour of footbridges under pedestrian loading. The Technical Department for Transport, Roads and Bridges Engineering and Road Safety, (2006).

3. P. Dallard, A.J. Fitzpatrick, A. Flint, S. Le Bourva, A. Low, R.M.R. Smith, The London Millennium Footbridge. Structral Engineer, 79, 22 (2001)

4. E.T. Ingólfsson. C.T. Georgakis, J. Jönsson, Pedestrian-induced lateral vibrations of footbridges: a literature review, Engineering Structures, 45 (2012)

5. S. Živanović, G. Feltrin, J.T. Mottram, J.M.W. Brownjohn, Vibration performance of bridges made of fibre reinforced polymer. IMAC-XXXII, Orlando, Florida, USA, 3 - 6 Feb. (2014)

6. C.A. Jones, P. Reynolds, A. Pavic, Vibration serviceability of stadia structures subjected to dynamic crowd loads: a literature review, Journal of Sound and Vibration, 330 (2011)

7. British Standards Institution, UK National Annex to Eurocode 1: Actions on structures-Part 2: Traffic loads on bridges. NA to BS EN 1991-2:2003 (2008)

8. A. Pavic, M. Willford, Vibration serviceability of post-tensioned concrete floors. Appendix G in Posttensioned concrete floors design handbook, Appendix G, Concrete Society, UK (2005)

9. Institution of Structural Engineers, Dynamic performance requirements for permanent grandstands: recommendations for management design and assessment (2008)

10. V. Racic, A. Pavic, J.M.W. Brownjohn, Experimental identification and analytical modelling of human walking forces: literature review. J Sound Vib, 326 (2009)

11. F. Venuti, L. Bruno, Crowd-structure interaction in lively footbridges under synchronous lateral excitation: a literature review. Phys Life Rev, 6 (2009)

12. C.J. Middleton, J.M.W. Brownjohn, Response of high frequency floors: a literature review, Engineering Structures, 32, 2 (2010)

13. S. Živanović, A. Pavic, P. Reynolds, Vibration serviceability of footbridges under human-induced excitation: a literature review. J Sound Vib, 279 (2005).

14. J.H. Rainer, G. Pernica, D.E. Allen, Dynamic loading and response of footbridges, Canadian Journal of Civil Engineering, 15, 1 (1988)

15. J.M.W. Brownjohn, A. Pavic, P. Omenzetter, A spectral density approach for modelling continuous vertical forces on pedestrian structures due to walking. Can. J. Civ. Eng., 31, 1 (2004)

16. L. Pedersen, C. Frier, Sensitivity of footbridge vibrations to stochastic walking parameters. J. Sound Vibrat., 329, 13 (2010)

17. S. Živanović, Benchmark footbridge for vibration serviceability assessment under vertical component of pedestrian load. Journal of Structural Engineering, 138, 10 (2012)

18. D.E. Newland, An introduction to random vibrations: spectral and wavelet analysis, Dover, New York. (2005)

19. E.T. Ingólfsson, C.T. Georgakis, J. Jönsson, F. Ricciardelli, Vertical footbridge vibrations: towards an improved and codifiable response evaluation, SEMC 2007, Cape Town, South Africa, 2007.

20. S.P. Carroll, J.S. Owen, M.F.M. Hussein, Modelling crowd-bridge dynamic interaction with a discretely defined crowd, J. Sound Vibrat., 331, 11 (2012)

21. K. Van Nimmen, G. Lombaert, I. Jonkers, G. De Roeck, P. Van den Broeck, Characterisation of walking loads by 3D inertial motion tracking, Journal of Sound and Vibration, 333, 20 (2014)

22. H.V. Dang and S. Živanović, Experimental characterisation of walking locomotion on rigid level surfaces using motion capture system, Engineering Structures, 91 (2015)

23. M.G. McDonald, Experimental characterisation of jumping and bobbing actions for individuals and small groups. PhD thesis, University of Warwick, UK (2015)

24. E.T. Ingólfsson, C.T. Georgakis, A stochastic load model for pedestrian-induced lateral forces on footbridges. Engineering Structures, 33, 12, (2011)

25. S. Živanović, A. Pavic, P. Reynolds, Probability based prediction of multi-mode vibration response to walking excitation. Engineering Structures, 29, 6 (2007)

26. V. Racic, J.M.W. Brownjohn, Stochastic model of near-periodic vertical loads due to humans walking. Advanced Engineering Informatics, 25, 2 (2011)

27. M. Bocian, J.H.G. Macdonald, J.F. Burn, Probabilistic criteria for lateral dynamic stability of 
bridges under crowd loading. Computers and Structures, 136 (2014)

28. C.C. Caprani, Application of the pseudo-excitation method to assessment of walking variability on footbridge vibration. Computers and Structures, 132, (2014)

29. J.H. Sim, A. Blakeborough, M.S. Williams, J.G. Parkhouse, Statistical Model of Crowd Jumping Loads. Journal of Structural Engineering, 134, (2008)

30. V. Racic, A. Pavic, Stochastic approach to modelling of near-periodic jumping loads. Mechanical Systems and Signal Processing, 24, (2010)

31. M. Duarte, S. Freitas, Revision of posturography based on force plate for balance evaluation. Revista Brasileira de Fisioterapia, 14, 3 (2010)

32. M.J. Griffin, Handbook of Human Vibration, Academic Press, London (1996)

33. P. Archbold, J. Keogh, C. Caprani, P. Fanning, A parametric study of pedestrian vertical force models for dynamic analysis of footbridges, EVACES 2011, Varenna, Italy (2011)

34. H. Geyer, A. Seyfarth, R. Blickhan, Compliant leg behaviour explains basic dynamics of walking and running. Proceedings of the Royal Society B, 273 (2006)

35. S. Kim, S. Park, Leg stiffness increases with speed to modulate gait frequency and propulsion energy. Journal of Biomechanics, 44 (2011)

36. D.J. Ewins, Modal testing: theory, practice and application, Research Studies Press, Baldock, 2000.

37. J.W. Dougill, J.R. Wright, J.G. Parkhouse, R.E. Harrison, Human structure interaction during rhythmic bobbing, The Structural Engineer, 84, 22 (2006)

38. H.V. Dang, Experimental and numerical modelling of walking locomotion on vertically vibrating lowfrequency structures, $\mathrm{PhD}$ Thesis, University of Warwick, UK (2014)

39. J.B.D.M. Saunders, V.T. Inman, V. T., H.D. Eberhart, The major determinants in normal and pathological gait. The Journal of Bone \& Joint Surgery, 53, 3 (1953)

40. S.A. Gard, D.S. Childress, What determines the vertical displacement of the body during normal walking? Journal of Prosthetics \& Orthotics, 13, 3 (2001)

41. H. Geyer, Simple models of legged locomotion based on compliant limb behavior, $\mathrm{PhD}$ thesis, FriedrichSchiller- Universität Jena, Germany (2005)

42. B.R. Whittington, D.G. Thelen, A simple massspring model with roller feet can induce the ground reactions observed in human walking. Journal of Biomechanical Engineering, 131, 1 (2009)

43. J.W. Qin, S.S. Law, Q.S. Yang, N. Yang, Pedestrianbridge dynamic interaction, including human participation. Journal of Sound and Vibration, 332, 4 (2013)

44. C. Barker, Some observations on the nature of the mechanism that drives the self-excited lateral response of footbridges, Footbridges 2002, Paris, France (2002)
45. J.H.G. Macdonald, Lateral excitation of bridges by balancing pedestrians. Proceedings of the Royal Society A, 465 (2009)

46. A.L. Hof, R.M. van Bockel, T. Schoppen, K. Postema, Control of lateral balance in walking: experimental findings in normal subjects and aboveknee amputees. Gait Posture 25 (2007).

47. A.D. Pizzimenti, F. Ricciardelli, Experimental evaluation of the dynamic lateral loading of footbridges by walking pedestrians. EURODYN 2005, Paris, France (2005)

48. E.T. Ingólfsson, C.T. Georgakis, F. Ricciardelli, J. Jönsson, Experimental identification of pedestrianinduced lateral forces on footbridges. Journal of Sound and Vibration, 330 (2011)

49. S.P. Carroll, J.S. Owen, M.F.M. Hussein, Reproduction of lateral ground reaction forces from visual marker data and analysis of balance response while walking on a laterally oscillating deck, Engineering Structures, 49 (2013)

50. M. Bocian, J.H.G. Macdonald, J.F. Burn, Biomechanically-inspired modeling of pedestrianinduced vertical self-excited forces. Journal of Bridge Engineering, 18, 12 (2013)

51. M. Willford, Dynamic actions and reactions of pedestrians. Footbridge 2002, Paris, France (2002)

52. J.M.W. Brownjohn, P. Fok, M. Roche, P. Omenzetter, Long span steel pedestrian bridge at Singapore Changi Airport - Part 2: Crowd loading tests and vibration mitigation measures. Structural Engineer, 82, 16 (2004)

53. S. Živanović, I.M. Díaz, A. Pavic, Influence of walking and standing crowds on structural dynamic performance. IMAC XXVII, Orlando, Florida, USA (2009)

54. J. de Sebastián, I.M. Díaz, C.M. Casado, A. Vasallo, A.V. Poncela, A. Lorenzana, Environmental and crowd influence on the dynamic behaviour of an inservice footbridge. Footbridge 2011, Wroclaw, Poland (2011)

55. L. Bruno, A. Tosin. P. Tricerri, F. Venuti, Non-local first-order modelling of crowd dynamics: a multidimensional framework with applications, Applied Mathematical Modelling, 35 (2011)

56. D. Helbing, A. Johansson, Pedestrian, crowd, and evacuation dynamics, in: R.A. Meyers (Ed.), Encyclopedia of Complexity and Systems Science, vol. 16, Springer, New York (2009)

57. F. Venuti, L. Bruno, Mitigation of human-induced lateral vibrations on footbridges through walkway shaping, Engineering Structures, 56 (2013) 